\title{
Robot Precise and Rapid Control PTZ PID Regulating
}

\author{
Bozhao Jiang ${ }^{1, a^{*}}$ and Chun Wang ${ }^{2, b}$ \\ ${ }^{1}$ Memorial High School, 935 Echo Lane. Houston TX USA 77024 \\ ${ }^{2}$ Association of Robot, China University of Petroleum, Beijing, 18 Fuxue Rd. Changping Zone, \\ Beijing China 102249 \\ a Jiang.bill123@gmail.com, b872866579@qq.com \\ * The corresponding author
}

\section{Keyword: PTZ; PID; Robot; MPU6050}

\begin{abstract}
This paper introduces the function and classification of PTZ, and takes the MPU-60X0 sensor and PTZ motor encoder as an example to carry out the research on the PID regulation mechanism of PTZ. Using the method of PID to adjust speed loop and position loop control to carry out the experimental parameters of rapid response of the robot. The experimental results show that the PTZ can keep the target position in the complex environment, and it has great anti - interference ability. The motor encoder makes the control angle more accurate, and the MPU-60X0 sensor PTZ is more stable, and the anti-interference ability of the PTZ is obviously improved. Encoder adjustment control makes control more accurate, but in fact, the PTZ reaction is slow, slow response to interference. The MPU-60X0 can make the PTZ has a great anti-interference ability. The intermodulation of the two adjustment methods is an effective way to make the PTZ work steadily.
\end{abstract}

\section{Introduction}

PTZ is the device to achieve the target object attitude control, the basic working principle is: send the command signal - the motor according to the instructions of rotation - the target object to achieve posture adjustment, the different PTZ device has the basically same mechanical structure, according to different control directions are generally divided into three: yaw axis control rotation; pitch axis control pitch; through can communication control. PTZ through the encoder feedback position for a circle $2^{13}$, in the middle there is a jump. Current effective range $-5000 \sim 5000 \mathrm{~mA}$.

MPU-60X0 is the world's first 9 axis motion processing sensor. It integrates 3 axis MEMS gyroscopes, 3 axis MEMS accelerometer and a scalable digital motion processor DMP (Digital Motion Processor), that connects a third-party digital sensor using the $\mathrm{I} 2 \mathrm{C}$ interface, such as a magnetometer. After expansion, you can output a 9 axis signal via its I2C or SPI interface (the SPI interface is available only on MPU-6000). MPU-60X0 can also connect non-inertial digital sensors via its I2C interface, such as pressure sensors.

The MPU-60X0 uses three 16-bit ADCs separately for the gyroscope and accelerometer, to convert the measured analog quantity to an output enable digital quantity. In order to accurately track the fast and slow motion, the sensor measurement range can be controlled by user, gyroscope measurable range is $\pm 250, \pm 500, \pm 1000, \pm 2000^{\circ} / \mathrm{s}$ (dps), accelerometer measurable range is $\pm 2, \pm 4, \pm 8, \pm 16 \mathrm{~g}$.

\section{PID Regulation Mechanism}

\section{PID is to Adjust the Deviation.}

$\mathrm{P}$ : proportional coefficient, directly plays a role on the deviation, $\mathrm{P}$ in the regulation of the speed play a major role, the greater the $\mathrm{P}$, the faster the speed, but the overshoot will be in a certain range with the increase, the General Assembly caused the shock.

I: integral coefficient, plays a role on the sum of deviation. It can eliminate the static difference, in practice with the role of $\mathrm{P}$ similar, because the two symbols in most of the time is the same. But the 
integral has a hysteresis effect, because the accumulation of points takes time, and it takes time to decrease after accumulating. The integral coefficient and the integral upper limit can be very different in different situations.

$\mathrm{D}$ : differential coefficient, plays a role on the difference of deviation, $\mathrm{n}$ fact, differentiation is the slope of the deviation change curve. The differential term has a leading effect. The system is controlled by the D-value between the time deviation and the last deviation, such as the change of deviation curve is intense, the effect of the differential item will be greater than that of the curve. And the differential term is the opposite of the sign of the integral term, so it is equivalent to reducing the output in the system. D can make the deviation change smoothly, but too large D will make the system sensitive.

The formula in the program can be briefly summarized as Eq. 1

$$
\text { Output }=P \times \mathrm{e}(t)+I \times \sum e(t)+D \times(e(t)-e(t-1))
$$

The method of adjusting the PTZ is the cascade PID adjustment, the inner ring is the position loop and the outer ring is the speed loop. The position can use the encoder angle or the MPU6050 angle. The output of the position loop is the target value of the speed loop. The speed loop output directly controls the input current of the PTZ. Speed loop can effectively reduce the interference in the environment, for the interference has an impedance effect, can make the PTZ smooth operation. The location allows the PTZ to reach the target location.

\section{PID Adjustment Speed Loop}

When the speed loop is adjusted, the position loop output is zero. Only to adjust the ratio of P, from small to large take $\mathrm{P}$. The approximate range of $\mathrm{P}$ can be an order of magnitude from the ratio of output to input. Now the maximum output of the PTZ is 5000, and the input of the MPU6050 is entered between 0 and 400 after the algorithm is modified. The maximum output to maximum input ratio is on the order of 10 orders of magnitude. So P started up from 10 . The greater the $\mathrm{P}$, the more stable the PTZ. The actual phenomenon can be manually disturbed by the senses. When in the hand shaking the PTZ, the greater the $\mathrm{P}$ the greater the resistance of $\mathrm{P}$, the more force the greater the resistance is also strong. According to the environment to choose a suitable $\mathrm{P}$ value as the speed loop of the tuning data, the other two items take 0 . Then adjust the position loop. According to the stability of the PTZ situation, fine-tuning speed ratio of $\mathrm{P}$.

\section{PID Adjustment Position Loop}

The adjustment method is generally adjusted the proportional term first, the other two items take $0, \mathrm{P}$ value from small to large, $\mathrm{P}$ value range can be based on the relationship between input and output to get the approximate range. Such as the encoder value range of $0 \sim 8196$, and the current output of $-5000 \sim$ 5000 , the two dimensions almost, so the value of $\mathrm{P}$ can take a class or ten class, without causing shock conditions as large as possible. Generally the second adjustment is the differential term, the differential coefficient $\mathrm{D}$ can be obtained by the desired target rate of change to get the approximate range of $\mathrm{D}$, you can also directly change the size of $\mathrm{D}$ to see the phenomenon, to determine the approximate range. The first rough adjustment and then fine. Integral items can eliminate the error, but the role of a long time, the role played in different systems have primary and secondary. In the two axes of PTZ control the yaw axis acts less effective, and the pitch axis is more effective.

Next, the effect of the parameters in the position loop adjustment is analyzed. 


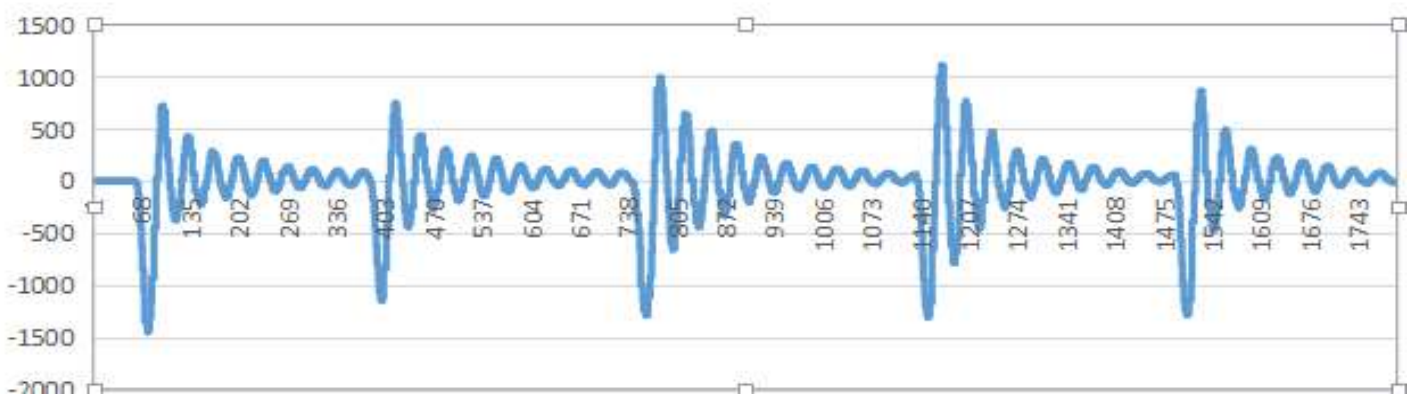

Figure 1. Parameters influenced by proportional coefficient $\mathrm{P}$

Fig. 1 shows the results of only the case of $\mathrm{P}$, in which case the system shock decreases gradually, but there are too many shocks cause a long time periods. At this time you can reduced P and also can add D. In this case it is recommended to add D.

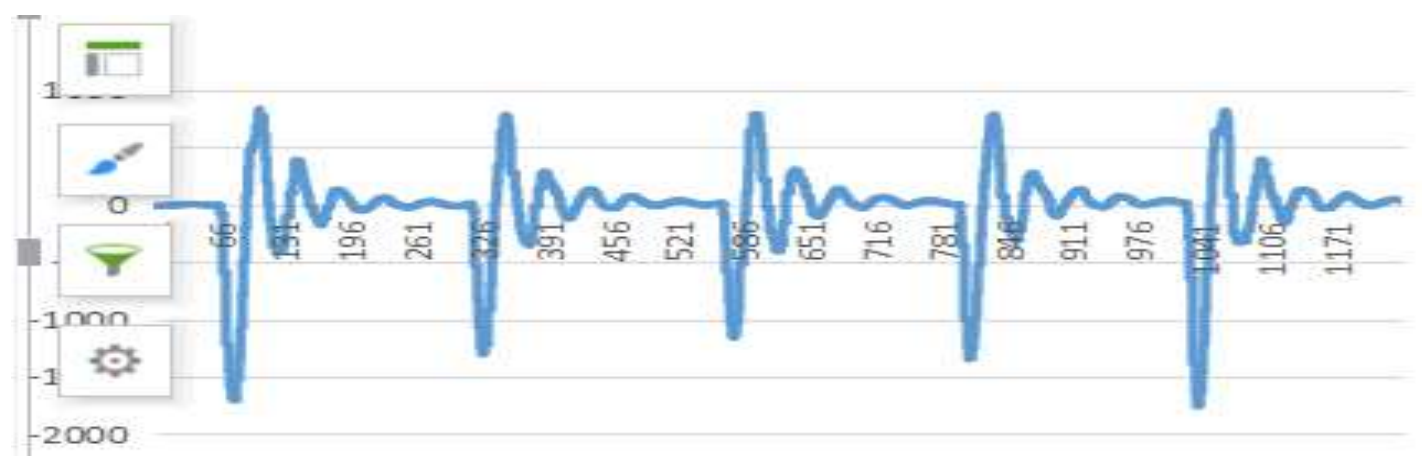

Figure 2. Parameters influenced by differential coefficient D

Fig. 2 shows the change after plus D, we can see that the slope of the change slowly, the number of shocks significantly less.

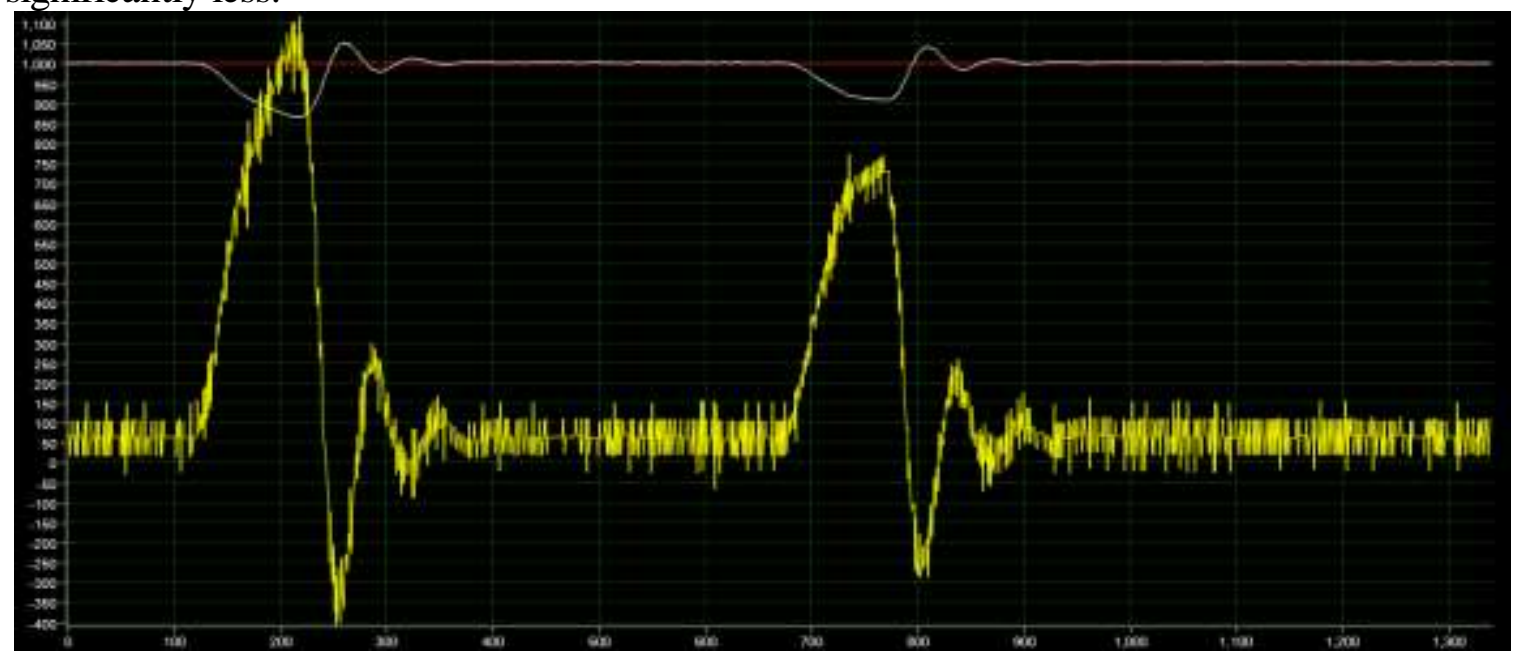

Figure 3. Parameters influenced by the differential term

Fig. 3 shows the ideal position change curve, the red line for the target value, white line for the curve. The yellow line is the output curve. With manual disturbance, first rise and then down, and finally stabilize at the target value. In the change of the yellow line, we can clearly see the effect of the differential term. When the overall rise and fall of the yellow line are stable, there are many burrs, which are the influence of the differential term on the rate of change, and then the change trend of the error is affected. 


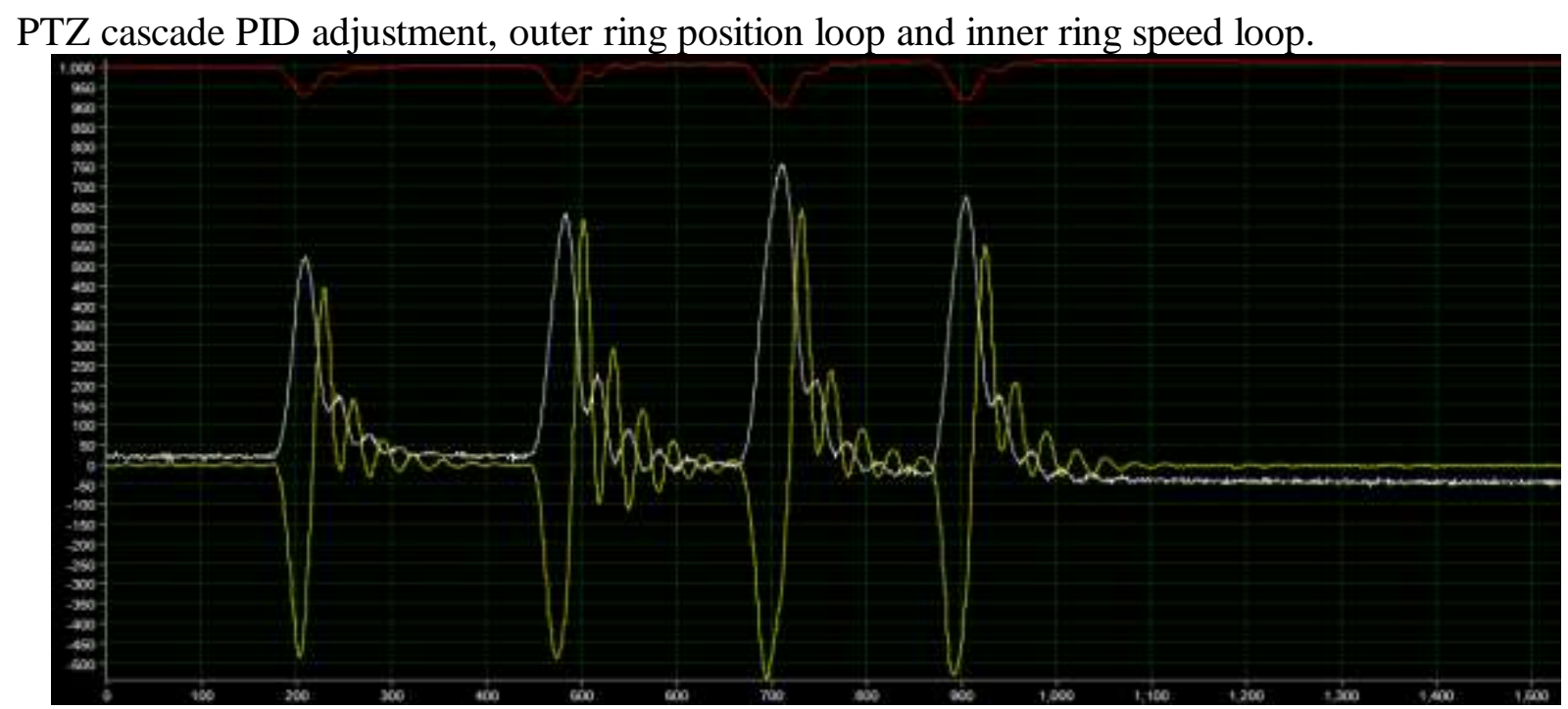

Figure 4. Position and speed change influenced by differential interference

Fig. 4 shows the position and speed changing which is influenced by differential interference. The red line is the position change curve, white line is the input change curve and yellow line is the speed loop output curve. As the fig. 4, The figure in the yellow line and white line changes seem to be the opposite, but in fact they should be the same, for the beginning is an enforced interference.

The above situation may be too high for the speed loop P or D is too small, it can be seen that the position change is slow and there is a situation away from the target, which is due to the actual detection of the speed is greater than the target speed. When the actual speed is greater than the target speed, the output negation causes the position to deviate from the target. The output is actually in order to approach the target, double loop control, the speed loop directly controls the current, That why it happened. The output of the position loop fluctuates up and down in the input, conforming to the actual situation, but it affects the stability of the position change. Lengthen the time to reach the target location.

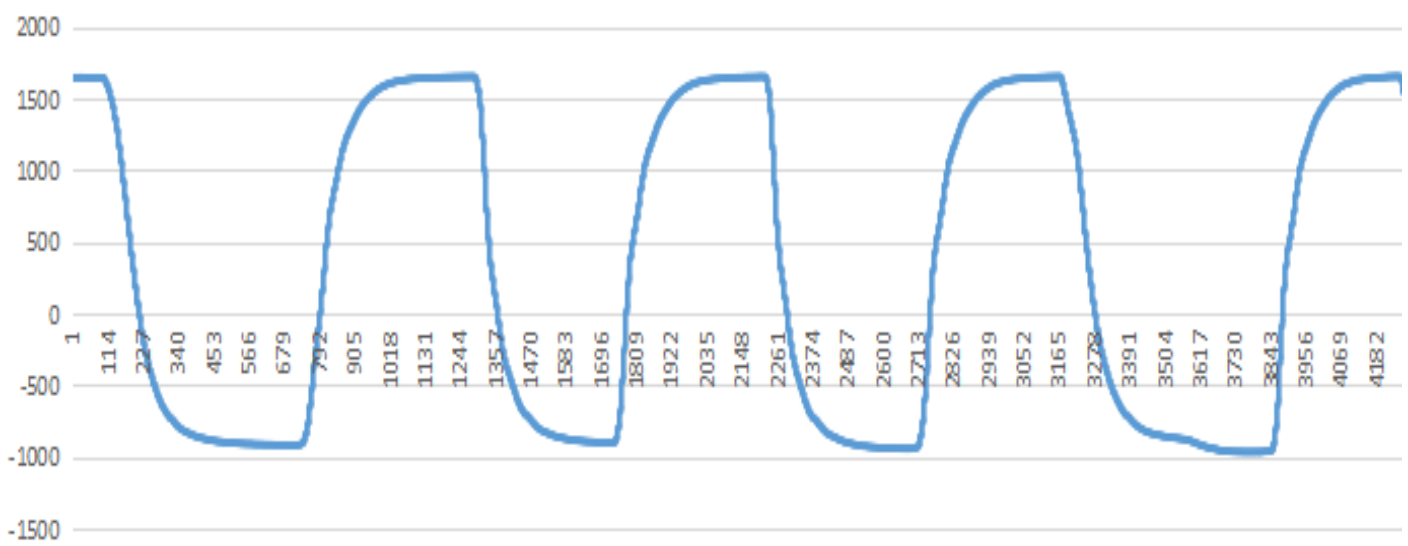

Figure. 5 Graph of the position change in the double-loop control

Fig. 5 shows the graph of the position change in the double-loop control, which is similar to the parameter adjusted for the previous graph, but this graph increases the value of $\mathrm{D}$ in the speed loop so that the actual speed is less than the target speed and does not cause a callback. The change is steady, but at close to the target value, the speed is greatly reduced, so that the time to reach the target value is greatly prolonged. 


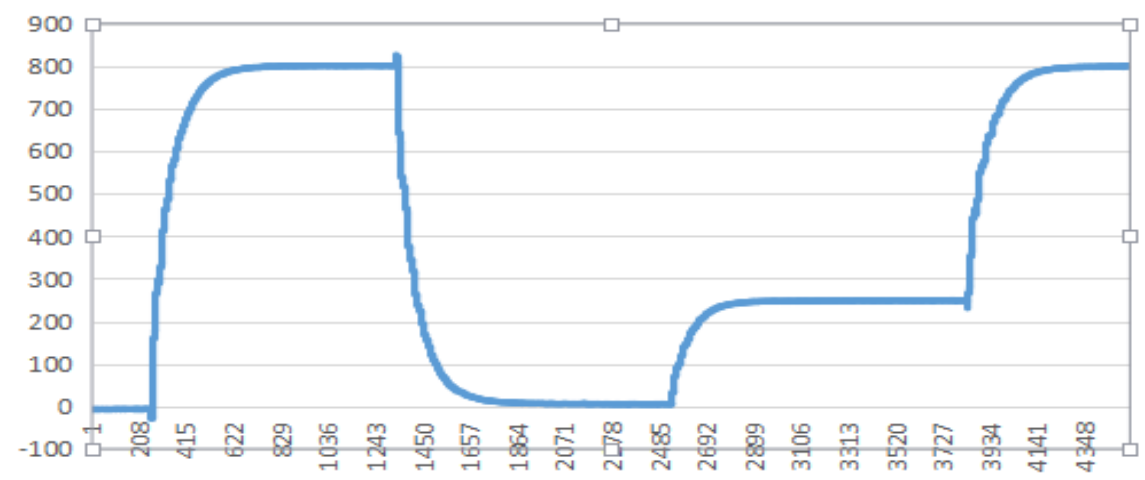

Figure 6. Ideal double loop position change curve obtained by experimenting

Fig. 6 shows the ideal double loop position change curve obtained by experimenting. By increasing the value of $\mathrm{P}$ in the position loop, the rate of change is fast and smooth. This can reduce the jitter of the PTZ when controlling through the remote control, or use the fixed-point position when the requirement of time is not high. To pursue faster speed, it is necessary to have a certain overshoot. Because the overshoot speed, change rapidly.

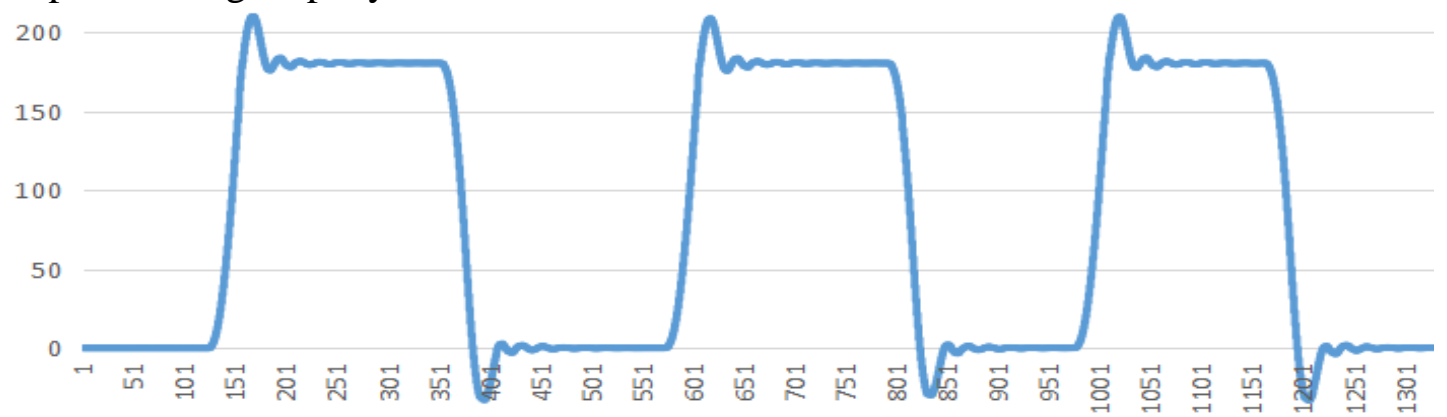

Figure 7. control curve obtained by limiting the maximum output of the speed loop and increasing the integral term

Fig. 7 shows the control curve is obtained by limiting the maximum output of the speed loop and by increasing the integral term. The first increases the value of I, the integral can be changed in a short period of time, and limit the integral upper limit, the general integral can gain the biggest role in the total output of about 1/5 (which is equivalent to increasing the scale effect), determined according to the actual situation. By comparing the results of the previous graph, the test results can reach the target value range in a short time, thus greatly reducing the robot's movement time.

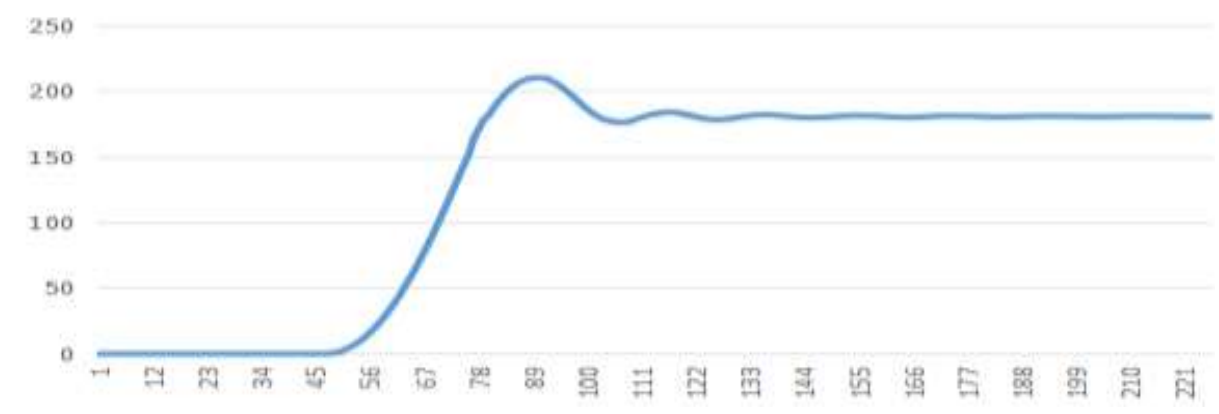

Figure 8. Partial enlarged view of the control curve in Fig. 7

Fig. 8 is a partial enlarged view of the figure, in which we can see that the control curve has already closed enough to the target value after the first overshoot. 


\section{Summary}

Through the study of the PID loop speed loop and position loop of the robot, I think that: the robot in the game environment is complex. The dramatic changes in speed, the collision of the robot, making the movement of the PTZ difficult to predict. So the actual debugging need to be adjusted independently. PTZ PID adjusts the speed loop, the position loop of the double loop control makes it easy to adjust independently. Independent adjustment of the PTZ and the robot as a whole is two separate individuals, PTZ in the movement for the robot in the environment more adaptable, and in the game is also by PTZ movement as the reference condition of chassis movement. The chassis following is a kind of excellent control method in PTZ control, which makes the robot control more flexible and easier to operate. So control the of the double ring is the basis for control of PTZ.

If you follow the "checklist" your paper will conform to the requirements of the publisher and facilitate a problem-free publication process.

\section{References}

[1] Wang Cheng-wen, Zhou Mei-wen, Guo Qing-ding. Vector Control AC Servo Motor (Mechanical Industry Press, China 1994).

[2] Shen Yong-fu. Summarization of Intelligent PID Control (China Machine Press, China 2002).

[3] Wang Li-xin. Fuzzy Control Tutorial M Paste System (Tsinghua University press, China 2003). 